\title{
Philosophiques
}

\section{Le plaisir esthétique et la vérité des sens}

\section{Herman Parret}

Volume 23, numéro 1, printemps 1996

Critères esthétiques et métamorphoses du beau

URI : https://id.erudit.org/iderudit/027368ar

DOI : https://doi.org/10.7202/027368ar

Aller au sommaire du numéro

Éditeur(s)

Société de philosophie du Québec

ISSN

0316-2923 (imprimé)

1492-1391 (numérique)

Découvrir la revue

Citer cet article

Parret, H. (1996). Le plaisir esthétique et la vérité des sens. Philosophiques, 23(1), 81-92. https://doi.org/10.7202/027368ar

\section{Résumé de l'article}

Certaines maximes énigmatiques dÉpicure nous parlent de l'essence du plaisir en termes de concentration et d'ex-centration, de condensation et d'ex- tension, d'implosion d'explosion. Un certain épicurisme chez Kant lui fait dire la même chose, là où le sentiment vital (Lebensgefùhl) est invoqué dans l'expérience esthé- tique. Le plaisir esthétique est mis en rapport avec la tensivité, le corps, la vie, le corps-en-vie. Toutefois, le plaisir, dans la tradition épicurienne, semble être l'inter- face de eros et aisthèsis, d'une érotétique et d'une esthétique, le corps-en-vie étant un corps-désir et un corps-sensation. Lucrèce, en bon épicurien et en préfi- gurant un certain Merleau-Ponty, évoque dans sa poésie géniale la vérité des sens, celle du corps-sensation défini à partir de la " sensation globale " du corps (synesthésie, interoceptivité). 


\title{
IE PIAISIR ESTHÉtIQUE ET LA VÉRITÉ DES SENS
}

\author{
PAR \\ Herman ParRet
}

\begin{abstract}
RÉSUMÉ:Certaines maximes énigmatiques d'Épicure nous parlent de l'essence du plaisir en termes de concentration et d'ex-centration, de condensation et d'extension, d'implosion d'explosion. Un certain épicurisme chez Kant lui fait dire la même chose, là où le sentiment vital (Lebensgefühl) est invoqué dans l'expérience esthétique. Le plaisir esthétique est mis en rapport avec la tensivité, le corps, la vie, le corps-en-vie. Toutefois, le plaisir, dans la tradition épicurienne, semble être l'interface de eros et aisthèsis, d'une érotétique et d'une esthétique, le corps-en-vie étant un corps-désir et un corps-sensation. Lucrèce, en bon épicurien et en préfigurant un certain Merleau-Ponty, évoque dans sa poésie géniale la vérité des sens, celle du corps-sensation défini à partir de la "sensation globale "du corps (synesthésie, interoceptivité).
\end{abstract}

ABSTRACT: Some enigmatic maxims of Epicurus discuss the essence of pleasure focusing on terms like concentration and excentration, condensation and extension, implosion and explosion. The epicurist tendency in Kant makes him saying similar things. He mentions "vital feeling "(Lebensgefühl) in his determination of the aesthetic experience. Aesthetic pleasure then is related to "tensivity", the body and life, the living body. However, pleasure, in the Epicurean tradition, seems to be the interface of eros and aisthèsis, of a erotetic and an aesthetic, because the living body is at the same time a body-desire and $a$ body-sensation. Lucretius, as a good Epicurean and announcing Merleau-Ponty, writes in his genial pom about the truth of the senses, the truth of body-sensation defined from the point of view of the "global sensation" of the body (synaesthesia, interoceptivity).

Comment Épicure, et Lucrèce à sa suite, en installant la règle du plaisir dans leur éthique, déterminent-ils l'essence du plaisir? Comment comprendre le plaisir qui accompagne la réalisation de tant de besoins hétérogènes ? Qu'y a-t-il de commun entre le plaisir d'être repu ou d'avoir chaud, le plaisir d'amitié ou de participer à l'ambiance communautaire, le plaisir de découvrir et de connaître, le plaisir de se sentir en bonne santé, le plaisir de l'écriture, le plaisir 
du felix aestheticus, de la vibration esthétique? Qu'en est-il du plaisir en tant que tel ? L'épicurisme nous enseigne la continuité de tous les plaisirs naturels, qu'ils soient nécessaires ou nonnécessaires. Toutefois, il semble que sur laxe des plaisirs il y a une rupture significative : il y aurait alors une classe de plaisirs qui, en se délivrant du besoin, conquièrent une autonomie à l'égard de désir, plaisirs ludiques plutôt, plaisirs gratuits. Ce serait là que se situent les euphories de l'expérience esthétique. Pradines, dans son Traité de psychologie générale, présente une hypothèse intéressante concernant cette émergence de la spécificité du plaisir esthétique, tout en exploitant à fond l'acquis épicurien'. Le plaisir, qui est originairement lié à une satisfaction du besoin-le besoin naturel et nécessaire, selon la catégorisation d'Épicure - peut se lier dans une phase plus évoluée aux sens $d u$ besoin. Ainsi l'odorat, le goût, n'importe quel sens peut anticiper la satisfaction. La sensation devient elle-mème une source de plaisir sans plus de référence directe au besoin : elle devient soi-disant "désintéressée ", comme dira Kant, source d'un "jugement de bon goūt ", voire d'une "connaissance sensible*. Sublimation sans doute, au moins symbolisation, "purification " bien suspecte aux yeux du psychanalyste, "esthétisation " de la sensation ludique rompant tout lien essentiel au désir. On est proche de la lecture kantienne du plaisir esthétique désintéressement, purification - et loin d'Épicure, dira-t-on. Il est vrai que le conatus de Spinoza, infiniment plus que le bon goút de Kant, se trouve à l'intersection d'Épicure et de Freud : tout vivant s'efforce de persévérer dans son ètre, c'est la pulsion d'autoconservation qui l'incline à fuir la douleur et à chercher le plaisir.

Mais mēme s'il y a sublimation et "purification " du plaisir dans l'expérience esthétique, il se trouve toujours qu'il y a un élément commun à tous les plaisirs, ce qui fait que le plaisir est plaisir, le moment essentiel du plaisir. Ramenons la question de l'essence du plaisir à un texte d'Épicure, texte mystérieux mais combien évocateur des énigmes du plaisir. La Maxime Capitale LX s'énonce ainsi :

Si tout plaisir se condensait avec le temps, et s'il ètait présent dans tout

lorganisme, ou dans les parties les plus importantes de notre nature, les plaisirs ne différeraient pas les uns des autres ${ }^{2}$.

Épicure évoque dans ce fragment le caractère non-différencié des plaisirs. Le plaisir est un quel que soit le sensible qui le provoque : le chant du rossignol tout comme le chant de la meilleure soprano du monde provoque du plaisir, le plaisir toujours "vécu " par notre corps comme non-douleur, par notre àme comme non-agitation. Mais cette

1. Voir en ce qui concerne Pradines, un article de M. Dufrenne, "Le plaisir esthétique *, dans Esthétique et philosophie, Paris, Klincksieck, 1981, III, p. 103-139.

2. Epicure, Maximes et Lettres, èd. M. Conche, Paris, PUF, 1967, p. 142. D'autres fragments sont également importants à cet égard, comme la Maxime Capitale $X I X$ : " Le temps infini contient un plaisir égal à celui du temps limité, si de ce plaisir on mesure les limites de la raison ". Voir aussi la Maxime Capitale XX: "La chair pose les limites du plaisir comme illimitées, et illimité est le temps qui le lui procure. Mais la pensée, qui s'est rendu compte de la fin et de la limite de la chair, et qui a fait disparaitre les craintes au sujet de l'éternité, procure la vie parfaite, et n'a en rien besoin, en plus, d'un temps infini [...]". 
non-différentiation des plaisirs est dépendante d'une condition qu'Épicure formule dans la Maxime Capitale $X^{3}$ à l'aide du terme de condensation (katepuknosis). On pourrait comprendre l'idée de la " condensation avec le temps " comme l'idée d'une totalisation, parallèle à la diffusion du plaisir dans l'ensemble du corps. L'intensité maximale du plaisir serait alors due à cette extension double: sur l'axe entier du temps - le présent devenant alors tripartite, comme le dira plus tard Augustin : le présent du passé, le présent du présent, le présent du futur - et sur le corps entier comme nœud de toutes les sensations. Le premier type d'extension ou de concentration a comme conséquence que le plaisir qui se trouve à son maximum, élargit le temps de la présence de sorte qu'aucun supplément de durée ne puisse l'accroître. Le plaisir est alors vécu comme Présence du présent/passé/futur, comme Permanence, comme Plénitude. De mème pour le second type d'extension ou de concentration : ce n'est plus tel ou tel organe spécifique, tel ou tel canal sensoriel isolé, mais le corps dans son unité qui "vit "le plaisir. Sous condition de cette double extension, on peut soutenir que le plaisir est non-différencié, que nous "vivons " ou bien le plaisir ou bien le non-plaisir, non pas un peu moins ou un peu plus de plaisir, non pas telle ou telle "qualité " de plaisir, non pas l'un ou l'autre "type "de plaisir. Cette interprétation de la Maxime Capitale IX nous a déjà fait progresser quelque peu. Si le plaisir non-différencié a le Temps Condensé comme ambiance et le Corps Concentré comme lieu, c'est que le plaisir est dans cette double mouvance de condensation d'une part et de dissémination de l'autre, d'intensification et d" "extensification ", de confusion et de diffusion. La plénitude d'un plaisir n'est pas sa perfection immobile et éternellement stabilisée, mais sa mouvance contradictoire de con-centration et de "ex-centration". Le plaisir dans sa plénitude implose et explose, tensivité constitutive du plaisir d'un corps-en-vie. Telle est la vérité du plaisir.

On a pourtant longuement discuté depuis Épicure la question de savoir si le mouvement est bien nécessaire pour qu'il y ait plaisir. Etre exempt de trouble et de douleur semble à première vue un plaisir pensé selon le repos (katastêmatikè). En effet, Cicéron, Sénèque, Plutarque, Augustin acceptent deux types de plaisir - le plaisir pensé selon le mouvement et le plaisir pensé selon le repos. Voici Augustin :

Les hornmes recherchent par nature ou le plaisir qui meut délicieusement par nature ou le repos qui fait que le corps ne souffre d'aucune gêne, tandis que l'un et l'autre sont appelés par Épicure du seul nom de plaisir ${ }^{4}$.

Cet embarras plane sur nombre d'interprétations des fragments d'Épicure. Cela ne peut ètre le cas que l'àme soit le siège des plaisirs en repos (katastêmatikè ; absence de souffrance et d'agitation) et le corps le siège des plaisirs en mouvement. Les plaisirs de l'àme seraient dans ce cas stables comme "un ciel sans nuages "tandis que les plaisirs de la chair diversifiés et fugitifs. C'est à peu près ce

3. Cette maxime hermétique a èté interprétée de plusieurs façons : voir $\mathbf{M}$. Conche, dans Épicure, Lettres et Maximes, Paris, PUF, 1987, p. 232-233. et G. Rodis-Lewis, Épicure et son école, Paris. Gallimard, 284 sqq.

4. Voir Rodis-Lewis, op. cit., p. 229-230. 
que Cicéron propose : l'élimination de la douleur (plaisir en repos) précède et commande lachèvement du plaisir qui atteint son terme (plaisir en mouvement) là où on peut le diversifier et l'amplifier. Et Cicéron reproche à Épicure, en fin de compte, d'avoir appelé ces deux types "incompatibles " de plaisir - le plaisir parfait et en repos, et le plaisir diversifié et en mouvement - par le mème nom. Pourtant Épicure est plus grand que Cicéron, et je prendrai sa défense. Il se pourrait, comme le pensait Aristote dans l'Éthique à Nicomaque, que le plaisir parfait et en repos ne soit pas une origine idéale qui se perd au cours de la mise-en-mouvement corporelle, mais bien plutôt un télos, un équilibre, une perfection, une plénitude immobile auquel le corps et ses plaisirs en mouvement aspirent. Le but ultime serait alors, pour l'âme comme pour le corps, le repos. Mais cette visèe téléologique, mème axiologique si l'on veut, ne changera pas le fait que l'élimination de la douleur lou plaisir catastématique, c'est-à-dire le plaisir en repos) n'est pas la condition sine qua non du plaisir en mouvement mais bien plutōt une conséquence, épiphénoménale dirais-je, du vrai plaisir qui est toujours en mouvement, qui est "vécu "dans sa double mouvance d'implosion et d'explosion, de concentration et d'ex-centration. C'est que la physico-anthropologie de l'épicurisme (mème si les textes d'Épicure lui-méme sont malheureusement fragmentaires à ce propos) donne un poids extrême et profondément constitutif au désir : c'est Eros qui génère le plaisir, n'importe quel plaisir dans sa concrétude émerge à partir d'un besoin érotétique. C'est le dynamisme du désir qui anime l'orientation première du vivant vers les satisfactions, et le plaisir est le nom d'une expérience contradictoire d'attraction et d'aversion, expérience nécessairement mêlée au sentiment de manque qui caractérise toujours le désir. La conception érotétique du corps-en-vie qu'est le vivant humain - conception spinoziste tout comme freudienne - ne supporte pas l'idée de deux types hétéronomes de plaisir. Il n'y a, quant à sa nature et son essence, qu'un seul plaisir, celui que nous devons de fond en comble à Vénus. Telle, encore, est la vérité du plaisir. Et Lucrèce, en bon épicurien, ne s'est pas trompé quand il ouvre son De Rerum Natura par un éblouissant hymne à Vénus :

Mère de la race d'Énée, joie des hommes et des dieux, Ô Vénus nourricière, toi qui, sous le ciel étoile où glissent lentement les astres, rends fécondes terre et mer, la mer qui porte les navires, la terre qui porte les moissons; toi en qui toute conception trouve son origine, toi par qui toute espèce vivante naît à la lumière du soleil, déesse. [...] C'est ton alliance, déesse, que je sollicite avec ardeur pour ces vers ${ }^{5}$.

C'est en ce lieu que, iconoclaste, j'ose introduire le sévère philosophe de Königsberg et le premier moment de sa détermination analytique du jugement de bon goût. En effet, le plaisir est le moment qualitatif de l'expérience esthétique, et c'est précisément dans ce contexte que Kant invoque explicitement la sagesse d'Épicure. La perspective érotétique (spinoziste) n'est évidemment pas envisagée par Kant - mais un certain épicurisme, pourtant, est puissamment

5. Je cite Lucrèce dans la traduction de Chantal Labre : Lucrèce, La nature des choses (De Rerum Natura), Édition Arléa, p. 17. 
présent : le plaisir (esthétique) est d'ordre corporel, le corps étant un corps-en-vie, un noud de forces vitales, un corps travaillé par les mouvements de stimulation et d'inhibition ${ }^{6}$ :

Le plaisir consiste toujours dans le fait que l'être humain ressent une stimulation de l'ensemble de sa vie, donc un accroissement de son bien-être physique, c'est-à-dire de sa santé ; dans cette mesure, Épicure, pour qui tout plaisir était au fond une sensation corporelle, n'a peut-être pas eu tort ${ }^{7}$.

Kant soutient, déjà au tout début de son Analytique, que l'expérience esthétique présuppose une "sensation de satisfaction " (Empfindung des Wohlgefallens) qui doit ètre rapportée au "sentiment vital "(Lebensgefühl) "que le sujet éprouve sous le nom de sentiment de plaisir ou de déplaisir ${ }^{8}$ ". Ce qui fait plaisir stimule également le sentiment d'ètre en bonne santé (das Gefühl der Gesundtheit) ${ }^{9}$ :

C'est tout le processus vital du corps (Lebensgeschäft im Körper) qui paraît se trouver, comme par un mouvement interne (als eine inner Motion), intensifié, ainsi qu'en témoigne l'excitation qui en résulte ${ }^{10}$.

On est en plein Épicure. Il y a, par conséquent, plus que la simple idée que " tout plaisir est en fait une sensation corporelle". En plus, le corps est vu par Kant tout comme par Épicure comme un "processus vital " que le plaisir esthétique intensifie " par un mouvement interne "de stimulation et d'inhibition, ou pour reprendre la terminologie épicurienne développée plus haut: par la double mouvance d'ex-centration et de concentration, de dissémination et de concentration, de diffusion et de confusion, d'explosion et d'implosion. Ainsi le plaisir est-il produit par un certain équilibre des forces vitales du corps. Par ailleurs, Kant illustre la structure de la double mouvance par l'exemple du rire, "affect procédant de la manière dont la tension d'une attente se trouve soudain réduite à néant ", le corps

6. Voici le passage entier : "Il est indiscutable aussi qu'en nous toutes les représentations, qu'elles soient objectives, simplement sensibles ou entièrement intellectuelles, peuvent être liées subjectivement au plaisir ou à la douleur, si imperceptibles qu'ils soient (toutes les représentations affectent le sentiment de la vie, et aucune ne peut ètre indiffërente pour autant qu'elle est une modification du sujet); il est mēme incontestable que, comme l'affurme Épicure, le plaisir et la douleur sont en fin de compte d'ordre corporel, peu importe qu'ils prennent naissance dans l'imagination ou dans des représentations de l'entendement, car, sans le sentiment de l'organe corporel, la vie serait la simple conscience de son existence, mais ne serait pas le sentiment de bien-être ou de malaise, c'est-à-dire le sentiment de la stimulation ou de l'inhibition des forces vitales. En effet, l'esprit n'est en luimême que vie (le principe vital lui-même) et les inhibitions et stimulations ne peuvent ètre cherchées qu'en dehors de lui, bien qu'en l'homme lui-même, et, partant, dans le rapport avec son corps". Kant, Critique de la faculté de juger, \$29, p. 277-278.

7. Ibid., \$ 54, p. 330-331. Le nom d'Épicure revient une troisième fois dans la Critique de la faculté de juger où Kant se répète quelque peu : "On peut donc, me semble-t-il. accorder à Épicure que tout plaisir, même si des concepts éveillant des idées esthétiques en sont l'occasion, est une sensation animale, c'est-à-dire corporelle *, \$54, p. 334.

8. CFJ, § 1, p. 204.

9. CFJ, \$ 54, p. $331-332$

10. CFJ, $\S 54$, p. 331. 
ressentant ce relâchement à travers l'oscillation des organes ${ }^{11}$ tensivité donc (at-tention et dètente), catégorie toute épicurienne que Kant met en scène sans en ètre trop conscient. Plaisir, corps, vie, tensivitê, voici une chaine conceptuelle qu'Épicure et Kant ont en commun. Plus est que, pour Kant comme pour Épicure, il est de l'essence du plaisir qu'il est indifférencié, indifférenciable: il n'y a ni degrés ni types de plaisir, il n'y a que plaisir ou non-plaisir. Kant, comme on sait, ajoute des critères supplèmentaires transcendant le pur sentiment du plaisir pour ètre en état de déterminer plus adéquatement, dans son "Analytique du Beau", la spécificité de l'expérience du beau. Le plaisir du beau, de l'agréable, de l'utile, le plaisir du corps ou de l'âme (Gemüt), c'est $d u$ plaisir, pure qualité. Les trois autres moments (surtout la quantité : est beau ce qui plaît universellement sans concept) nous font sortir, de toute évidence, du cadre épicurien. Épicure a été pour Kant une bonne heuristique pour l'élaboration conceptuelle de ce qu'il pensait être le plaisir comme qualité, ce qui ne fait pas encore, nul n'en doute, une Critique de l'appréciation esthétique ${ }^{12}$. Il faudra bien d'autres pierres pour achever la Cathédrale.

Plaisir. corps, vie, tensivité, telle est la chaine commune à Kant et Épicure. Mais ce corps-en-vie soumis à la double mouvance tensive, de quel corps s'agit-il ? Kant ne considère jamais le corps dans sa profondeur, le corps-désir, le corps des besoins naturels (nécessaires et non-nécessaires) tandis que, de Spinoza à Freud, c'est dans ce corps, érotétique, que s'incrustent les plaisirs. Opposée à cette conception "verticale "de la corporéité, il y en a une autre, l" horizontale ", le corps dans son extension, le corps-sensation, le corps des cinq sens et de leur coordination. Eros d'une part, aisthèsis de l'autre, érotétique et esthétique, le corps-en-vie profite des deux, de ses dēsirs en profondeur, de ses sensations en surface. Et le plaisir, dans son essence indiffërenciée, est tributaire des deux registres: c'est que le plaisir est l'interface d'eros et d'aisthèsis, d'une érotétique et d'une esthétique. Il y aurait donc deux types d'affection : on est affecté par ses désirs ou on est affecté par ses sensations. Et il y a, par conséquent, deux tendances de structuration des plaisirs : ou bien l'érotétisation des plaisirs ou bien l'esthétisation des plaisirs. L'épicurisme - il y a évidemment plusieurs lectures d'Épicure,

11. CFJ, § 54, p. 332 . Voir également, pour des considérations kantiennes dans les mêmes termes concernant le rire et les larmes, l'Anthropologie du point de vue pragmatique, \$ 79, p. 261-262.

12. On peut retoumer au Livre II de l'Anthropologie du point de vue pragmatique de Kant pour trouver, en termes bien simples, la division suivante du Sentiment de plaisir et de déplaisir : "A. Le plaisir sensible, B. Le plaisir intellectuel Le premier est présenté ou bien A. par les sens le consentement), ou bien B. par l'imagination (le goùt); le second (à savoir l'intellectuel) l'est ou bien A. par des concepts susceptibles d'être présentés, ou bien B. par des Idées », § 59, p. 229. C'est le point de vue toujours pragmatico-psychologique qui élargit la portée du plaisir jusque dans le domaine intellectuel de la conceptualisation et de l'Idéalisation. Le point de vue transcendantal dans la Critique de la faculté de juger limitera de manière stricte l'impact du plaisir ; sans plaisir, pas d'appréciation esthétique (c'est le moment de la qualitè), c'est une condition nécessaire mais pas suffisante : pour qu'il y ait appréciation esthétique, il faut en plus les trois autres moments de l'Analytique du Beau, surtout le moment quantitatif de l'exigence d'universalité. 
surtout parce que l'on ne dispose que de quelques lettres, maximes et fragments du philosophe - tend à privilégier la perspective "verticale ", un certain fondamentalisme des profondeurs qui mènera à Spinoza et à Freud : le plaisir est dans la satisfaction d'un besoin. Il est dit alors que ce qui émeut, caresse et flatte la sensibilité est superficiel, instable et complémentaire. L'essentiel est alors que tous les désirs naturels et nécessaires soient comblés. La demande esthétique des cinq sens-fugitivité, raffinement, fragilité des caresses, des goūts et des regards - est dite inciter à la débauche, à l'inessentiel. Cette tendance à l'érotétisation des plaisirs consacrerait en fait les seules nécessités vitales. L'autre paradigme - et on frôle Kant, ou moins le Kant de la Critique de la faculté de juger proclame que les plaisirs sont dans les sensations, dans la vie sensitive des cinq sens. Ce qui compte alors est la richesse " horizontale" des sensibles, la fleur de peau des surfaces, la chair du monde, les timbres et chromies, le jeu des sensations. C'est la tendance à l'esthétisation des plaisirs: le sensible exerce sa séduction, le comble des plaisirs est dans l'ivresse sensorielle du felix aestheticus. Le corps, de toute évidence, est le substrat nécessaire dans les deux perspectives, corps-désir ou corps-sensation, deux figures du corps-en-vie. Et la double mouvance qui caractérise le processus vital - concentration et ex-centration, implosion et explosion - remplit ces deux figures de manière bien spécifique. Les plaisirs "en mouvement " du corps-désir sont pulsionnels - Épicure, Spinoza, Freud - tandis que les plaisirs " en mouvement " du corpssensation sont ludiques : comme l'explique Kant, c'est, dans ce cas, le jeu des facultés (l'entendement et l'imagination surtout: la façon dont on transpose la sensation par rationalisation et symbolisation, dans le rêve et le phantasme, dans la fiction) qui provoque la tensivité bien ludique des plaisirs. Épicure et Kant, de belles fiançailles, il est vrai, mais combien imparfaites. Le plaisir entre le désir et la sensation Épicure et Kant, pourtant, poussent cette balançoire dans des directions passablement déviantes.

De Rerum Natura (La nature des choses), la "sémiotique lucrétienne ", est un Cantique des Cantiques jubilant les gloires de la sensation, les fastes du sensible. Le Livre IV sur les illusions et phantasmes de l'âme, sur les simulacres et les songes, comporte une sémiotique admirable de la vie sensorielle à partir d'une analytique des virtualités des cinq sens. Lidée du simulacre et de l'illusion référentielle a été largement exploitée en sémiotique contemporaine mais elle trouve son précédent le plus lumineux dans Lucrèce. Le poète-philosophe décrit finement ces simulacres et leur physique tandis que le livre dans son entièreté est une puissante apologie des cinq sens constituant la vie sensitive de l'ètre humain, ce corps-envie qui jouit et qui souffre. La vérité des sens "n'impute pas à la vue les erreurs de l'esprit ${ }^{13}$, ni aux autres sens. En enlevant d'une part

13. Lucrèce, p. 163. Encore : "Nous n'accordons pas pour autant que les yeux puissent se tromper le moins du monde. Voir, où elles sont et où qu'elles soient. l'ombre et la lumière sont ou non les mềmes, si c'est la même ombre qui, tout à l'heure ici. est passée là-bas... cela. c'est à la raison, à l'esprit de le discerner: il n'est pas au pouvoir des yeux de connaitre la nature des choses. N'impute donc pas à la vue les erreurs de l'esprit *. 
les opinions (doxa) et les passions (dans les termes d'Épicure : les désirs non-naturels) de l'autre, on retrouve la vérité de la vie sensorielle, ses gloires et richesses sollicitées par les fastes du sensible. Tout comme le sentiment du plaisir est toujours un bien, de même la sensation est toujours vraie. Et je cite un long passage où Lucrèce développe sa thèse concernant la vérité des sens.

Tu trouveras que ce sont les sens qui, d'abord, nous ont donné la notion du vrai, et comprendras que les sens ne peuvent ètre réfutés. [...] Gui est plus digne de confiance que les sens ? [...] S'ils nous trompent, la raison tout entière n'est, elle aussi, qu'erreur. Mais peut-être penses-tu que l'oü̈ va rectifier les défauts de la vue, le toucher ceux de l'oüe ? Gue le goūt, peutètre, va dénoncer l'erreur du toucher, que l'odorat réfutera le témoignage des autres sens ou que la vue les convaincra d'erreur? Je n'en crois rien : chaque sens a son pouvoir propre et son domaine réservé. La sensation du mou, du glacé, du bouillant, est donc nécessairement bien distincte de la perception des diverses couleurs et de tout ce qui se rattache à la notion de couleur. Le goût a lui aussi son univers propre ; l'odorat fonctionne de son côté comme l'ouïe du sien. Il est aussi absolument impossible que les divers sens puissent se réfuter les uns les autres [...] : ce qu'ils perçoivent, et à n'importe quel moment, est vrai ${ }^{14}$.

En ce qui concerne le statut de la sensation (aisthèsis), Lucrèce ne diffère guère d'Épicure. Le fait de ressentir fait jaillir l'évidence. La sensation est critère de vérité (de nos pensèes sur la réalité) parce que, en deçà de la pensée, elle est ce par quoi il y a pour nous vérité, c'est-à-dire accès à la réalité. Dans le domaine de la connaissance comme dans celui de l'affectivité, la sensation n'a pas besoin de justification pour celui qui sent. La sensation est ce à partir de quoi la raison justifie, mais elle-même n'a pas besoin de preuve : elle est "irrationnelle "(alogos), muette. Le sensible est exactement comme il apparait. La sensation n'ajoute rien, elle n'a rien de subjectif et de construit : elle se situe d'ailleurs à un niveau où il n'y a pas encore de " sujet ". La sensation est infaillible et l'évidence sensorielle a une valeur absolue. Il ne saurait donc y avoir la moindre différence, à cet égard, d'un sens à un autre, ou, pour le mème sens, d'une sensation à une autre, car vouloir corriger les sensations les unes par les autres - celles de la vue par celles de l'ouïe, celles de l'ouie par celles du toucher, etc. - serait admettre la possibilité de sensations erronées, ce qui serait ruiner le fondement de la connaissance.

Et Lucrèce traite par la suite les cinq sens séparément en ce qu'ils ont de plus poétiquement spécifique. Avec le même sensualisme que l'on retrouvera vingt siècles plus tard dans ce grand lucrétien qu'est le Greimas de De l'imperfection. Mèmes enthousiasmes, mèmes finesses, mèmes sensitivités. Prenons ainsi l'ouïe - évocation de la rudesse et de la douceur des sons, de la tonalité des voix qui déchirent la gorge et l'oreille, des musiques, ainsi que les "douces plaintes de la flûte ", des échos que les collines se renvoient les unes aux autres spontanément, le chant du coq qui met jusqu'au lion en colère. Ensuite les goûts et ses organes - langue et palais, la saveur qui envahit la bouche, les salives, les sucs qui se rêpandent dans la 
gorge, les vins qui chatouillent les pores du palais, les vinaigres qui déchirent le gosier. Et les odeurs. "Comment viennent-elles effleurer nos narines ? [...] Comment l'odeur du miel attire-t-il de loin l'abeille, celle des cadavres les vautours? ${ }^{15}$ ". Et Lucrèce de décrire l'impact spécifique des odeurs et saveurs, des images et des couleurs sur l'esprit : certaines sensations comme les odeurs * flottent au hasard, arrivent tard, s'éteignent quelquefois avant d'ètre sensibles, se dissipant peu à peu dans les airs ${ }^{16}$ ", fugitivité à opposer à la permanence des images - certains sens sont plus objectivants, d'autres plus intimes. Esthétique de notre corps-en-vie aux cinq sens, "vérité " complexe, subtile des sens, surplombée, affectée par une réalité affluente, inépuisable " en horizontale ", une réalité de surfaces qui s'étendent jusqu'à l'infini. Cependant, on l'a vu, ce corps-en-vie qu'est l'âme semble concerné par plus que la simple sensation totalement soumise et passive à l'égard de l'll y a sensible du réel ${ }^{17}$. Et c'est à ce point que l'esthétique lucrétienne devient intéressante. En fait, l'āme contient plus que des sensations produites par un seul canal sensoriel isolé. Lucrèce évoque - sans trop approfondir, il est vrai - l'idée que les sensations qui se succèdent ne sont pas perçues séparément, isolément, mais nous font "percevoir "la chose dans son intégralitè. Je cite le passage où le poète-philosophe lui-mème introduit le concept de sensation globale.

Guand le vent nous cingle sans relâche, quand un froid terrible s'insinue en nous, nous ne sentons pas non plus chaque particule de vent ou de froid: nous n'avons qu'une sensation globale: nous sentons notre corps tout meurti à l'intérieur, comme si quelque choc le frappait de l'extérieur et nous donnait le sentiment de la réalité. Un autre exemple: si nous frappons du doigt contre une pierre, ce que nous touchons, alors, c'est sa surface et la couleur émise par cette surface : ce n'est pourtant pas cela que le toucher nous fait sentir : la sensation qu'il nous apporte, c'est bien plutôt celle de la dureté essentielle qui réside dans les profondeurs du bloc ${ }^{18}$.

15. Lucrèce, op. cit., p. 172

16. Lucrèce, op. cit., p. 173 .

17. L'âme contient, à part les sensations, également les affections (pathè) et les anticipations (prolèpsis) qui évidemment ne valent que par les sensations auxquelles elles renvoient. Pour Épicure comme pour Lucrèce, il n'y a qu'un seul critère essentiel : la sensation, mais, pour une analyse exhaustive, il faut déterminer également les deux autres critères de la canonique épicurienne. L'affection : le plaisir et la douleur, "réponse immédiate " sitôt ressentie, d'une part, et l'anticipation, concept difficile mais extrêmement fructueux et moderne. Il s'agit du processus d'abstraction selon Épicure : une mēme sensation, se répétant dans des contextes sensoriels différents, se trouve comme " abstraite " par le fait même. Elle laisse dans la mémoire une empreinte distinctive qu'un mot peut évoquer. Ce " concept * - présent dans le mot désignant la sensation-commence alors à signifier des sensations possibles. Tout discours humain ne vaut. par ailleurs, que par l'éventualité d'un remplissement sensoriel. S'il n'y avait les sensations, il n'y aurait pas de langage. Et nous pouvons user de mots parce que les sensations auxquelles ils renvoient ont chance de réapparaitre. Les anticipations peuvent "vivre leur propre vie ", détachées des sensations. La prolèpsis considérée du point de vue de la psychologie cognitive contemporaine, est une " trouvaille " épistémologique très originale de l'épicurisme.

18. Lucrèce, op. cit., p. 159. Voir également, P. Boyancé, Lucrèce et l'épicurisme, Paris, PUF, 1963. 
Sensation globale, donc: " nous sentons notre corps tout meurtri à l'intérieur . Intéroceptivité, ou sensation du corps comme centre de coordination des sensations. "Toucher une couleur émise par les surfaces *, synesthésie parmi les plus difficiles : toucher le visible. Il y a, par conséquent, chez Lucrèce une certaine idée du corps intégral dont le " sens intérieur " génère des synesthésies. Idée, on n'en doute pas, d'une extrème modernité, qui corrige quelque peu la portée du texte de Lucrèce cité il y a un instant : "[...] que chaque sens a son pouvoir propre et son domaine réservé ". Il se révèle en fait que, pour Lucrèce, toute sensation résulte d'un contact: les sensations "rendent tous les objets des sens touchables (hapta) et, s'il en est ainsi, chacun des autres sens est une sorte de toucher. La synesthésie trouvera ainsi son explication : il y aurait un toucher fondamental dans lequel se rassemble toute virtualité sensorielle. Je cite Greimas, ce lucrétien bien contemporain, qui exprime la même intuition dans De l'imperfection:

Le toucher, la plus profonde des sensations à partir desquelles se développent les passions du corps et de l'àme, vise, en fin de compte, la conjonction du sujet et de l'objet, seule voie menant à l'esthesis. [...] Le toucher est plus que l'esthétique classique veut bien lui reconnaitre [...] il se situe parmi les ordres sensoriels les plus profonds, il exprime proxémiquement l'intimité optimale et manifeste, sur le plan cognitif, le vouloir de conjonction totale ${ }^{19}$.

Point de vue exemplairement lucrétien. Si l'on veut préciser la nature de l'évidence sensorielle, il faut la définir comme évidence tangible : sentir, c'est toucher. Le réel, en effet, est ce qui se fait sentir, le réel lui aussi est corporel. Et la sensation, par conséquence, est dans le choc des corps : corps du monde, corps-en-vie. Or le rapport immédiat d'un corps et d'un autre corps est le contact. Comment dire chose pareille de l'odorat, de l'ouie, de la vue, sens soi-disant "à distance "? Pour Lucrèce, le contact, il est vrai, n'est pas avec les objets mèmes, mais avec leurs émanations - c'est la théorie des simulacres, développée précisément dans le Livre IV, "Simulacres et illusion ", de La nature des choses. L'image que nous avons de l'objet est le résultat d'un contact direct, même si les simulacres peuvent être déformés, déchirés, rongés par les milieux traversés. La thèse de la prépondérance du toucher devrait intéresser la sémiotique comme une thèse extrêmement pertinente, même si l'atomisme et le matérialisme de Lucrèce ne nous paraissent guère acceptables.

Surgissent en ce lieu les fiançailles, inattendues sans doute, de Lucrèce et de Merleau-Ponty. Cadre matérialiste d'une part, et phénoménologique de l'autre, mis à part, il y a une esthétique où Lucrèce et Merleau-Ponty se rencontrent. C'est que les deux philosophes expliquent la synesthésie et la " sensation globale "intéroceptive de la chose du monde - que Merleau-Ponty qualifie de chair (puisque le monde est fait de chair tout comme le corps-en-vie) - par la dominance $\mathrm{du}$ toucher fondamental ${ }^{20}$. Merleau-Ponty, dans la Phénoménologie de la perception, parle volontiers de la communi-

19. A. J. Greimas, De l'imperfection, Perigueux, Fanlac, 1987, p. 92 et 30.

20. Voir mon article "Synesthésies du visible", dans Versus, 65/66, 1993 , p. 59-70. 
cation entre les données des divers sens : la relation intersensorielle de communication est possible à cause de l'unité première du sentir. Selon Merleau-Ponty, l'articulation du système sensoriel en cinq sens présuppose un fond indifférencié où il y a confusion des sens : la vision, ou un autre sens, n'y est pas encore assez fortement installée pour exercer un rōle articulé. C'est ainsi que Merleau-Ponty a pu écrire que "la fragilité, la rigidité, la transparence et le son cristallin d'un verre traduisent une seule manière d'être ${ }^{21}$ ". L'unité de la chose est pré-esthésique, et c'est le sentir confus qui est capable de récupérer cette unité mondaine et réelle, appelée chair, ce senti d'avant les sens isolés, d'avant la différenciation sensorielle. La manière d'être du verre, l'unitè de la chose, se laisse traduire alors en mème temps en son, en image, en "tangème". La perception est Einfühlung, sympathie avec la chair du monde, puisque mon corps lui aussi est taillè dans la tissure du sensible. Le visible et l'invisible situe bien, évidemment le sentir primordial dans le toucher, le contact tactile $^{22}$. Le toucher est l'origine de la sensibilitè entière et donc, de tout sens. Le regard tout comme la voix, la vue tout comme l'ouie, "touchent". Les synesthésies, phénoménologiquement, font remonter les sensations, produites par les canaux sensoriels spécifiques, vers leur origine commune, le pré-esthésique, le toucher fondamental.

Gloires de la sensation : la diversité et la richesse de notre vie sensorielle s'organisent, s'hiérarchisent autour du toucher fondamental, le contact avec le réel. Fastes du sensible: ce réel est une présence inépuisable qui se laisse toucher, qui se donne à toucher par nos cinq sens. Le touchant et le touché, dans leur réversibilitè radicale, c'est l'inter-corps, la chair du monde dont est faite aussi la chair de notre corps-en-vie. Cette esthétique-là est la source intarissable des plaisirs. Toutefois, le plaisir, on l'a suffisamment accentué, est l'interface d'une érotétique et d'une esthétique, interface des besoins et des sensations. Et le corps-en-vie est le nœud du corpsdésir et du corps-sensation. Le plaisir, c'est la vérité des besoins tout comme la vérité des sens. Tel, en tout cas, est l'enseignement d'Épicure, si conséquemment transposé dans le poème philosophique de Lucrèce. Transposé encore, de façon testamentaire, dans le message de Greimas. La sémiotique lucrétienne, en fin de compte, nous rappelle, mais avec quel gènie, que l'être-dans-le-monde et

21. M. Merleau-Ponty, La phénoménologie de la perception, Paris, Gallimard. 1945 , p. 368.

22. "Avec la réversibilité du visible et du tangible, ce qui nous est ouvert, c'est donc, sinon encore l'incorporel, du moins un ètre intercorporel ", Le visible et l'invisible, p. 188. "Mes mouvements ne vont plus vers les choses à venir, à toucher, ou vers mon corps, en train de les voir et de les toucher, mais ils s'adressent au corps en général et pour lui-mème ", Ibid., p. 189. Pour le chiasme chair du monde/chair du corps, voir les Notes de travail, p. 302303 , et surtout 307-310: kToucher-se toucher, voir-se voir, le corps, la chair comme Soi n. Il est êvident que le métalangage de Merleau-Ponty est à l'antipode de celui de Lucrèce, et la phénomenologie peut même ètre considérée comme une attaque en règle contre la physique matérialiste et atomiste de Lucrèce. Toutefois, les a fiançailles " ne concernent pas le métalangage mais l'acceptation commune des phénomènes de synesthésie et d'intéroceptivitè comme ètant fondamentaux pour l'esthètique lou la doctrine des sensations). 
l'être-avec-les-autres est un intercorps de besoins et de sensations dont le plaisir nous dit la vérité.

Fonds National belge de la Recherche Scientifique

Universités de Louvain et d'Anvers 